\title{
Convergence of the Vortex Filament Method
}

\author{
By Claude Greengard*
}

\begin{abstract}
Fully discrete convergence estimates have previously been given for the three-dimensional vortex method proposed by Beale and Majda. It is shown in this paper that vortex filament methods of the kind used in practice converge, provided smooth vortex structures consisting of closed filaments are appropriately discretized, and the stretching of the discrete filaments is computed sufficiently accurately. The error estimates obtained are those of the previous theory.
\end{abstract}

1. Introduction. Many interesting numerical experiments have been carried out over the past decade by various authors in which three-dimensional, incompressible, inviscid fluid motions are calculated by vortex filament methods. In these methods initial fields of vorticity are approximated by one or more discretized vortex filaments; the evolution in time of the filaments is determined by computation of the interactions of these filaments with one another and with themselves. Leonard has recently surveyed numerical experimentation with three-dimensional vortex methods [11].

A convergence theorem for the filament method is presented in this paper. It is shown that if sufficiently smooth vortex structures consisting of closed filaments are appropriately discretized, and the stretching of vorticity is computed accurately, then the particle trajectories which are calculated in the algorithm converge to the exact trajectories as the spatial and temporal discretization parameters go to zero. The analysis given in this paper suggests appropriate choices for weight factors and vortex stretching formulas in the filament method, the adoption of which leads to convergent schemes. We note that it follows from the convergence of the trajectories that the computed velocity and vorticity converge (the latter in a weaker norm, however) as well.

The statement of the convergence theorem and its proof are closely related to the paper by Anderson and Greengard [1], which in turn owed much to the convergence theory of Beale and Majda [2], [3]. Beale and Majda gave the first convergence proof for a three-dimensional vortex method, following Hald's convergence proof [9] for the planar vortex method. A major difficulty in the analysis of three-dimensional vortex methods, not present in the two-dimensional case, is the necessity of estimating the errors introduced in the vorticity by a numerical approximation of vortex

Received March 11, 1985; revised February 21, 1986.

1980 Mathematics Subject Classification. Primary 65M15; Secondary 76C05.

*Current address: IBM Watson Research Center, Yorktown Heights, New York 10598. 
stretching. This difficulty was very nicely overcome by Beale and Majda [2], [3], who studied an algorithm of their own design in which the initial fluid particle positions are the nodes of a cubic mesh and in which derivatives in time of the vorticity are evaluated by application of a centered finite-difference operator (with respect to the Lagrangian variables) to the computed velocity field. A stability error bound on the velocity was obtained involving, in part, the discrete Sobolev $H^{-1}$-norm of the error in vorticity, which is converted into an $H^{0}\left(=L^{2}\right)$-norm of the error in the flow map. Semidiscrete (spatial, but not temporal, discretization) convergence estimates were obtained of arbitrarily high-order accuracy using this stability result and a consistency lemma. The consistency results of [2], [3] were strengthened and simplified in [1] (following work of Cottet [6]). Fully discrete convergence estimates, which follow directly from the stability and consistency lemmas, were also given in [1].

The filament method, in which the fluid particles whose trajectories are calculated lie along vortex filaments, is in general more natural to implement and reveals vortex structures better than the method studied in [2]. The vectors of vorticity used in the calculation of the particle velocities in the filament method are evaluated by computation of the separation of neighboring particles along the filaments. It was pointed out in [1] (see also the example in Section 2 below) that this amounts to the evaluation of vortex stretching by the application of a Lagrangian finite-difference operator to the set of particle positions. A fortunate consequence of this fact is that modifications of the ideas in [1], [2], [3], [6] can be used to obtain a convergence proof for the filament method. We describe now briefly the main ideas of the proof.

The convergence of a three-dimensional vortex method requires that the singular integral which expresses the velocity as a function of the vorticity be accurately discretized, that the stretching of vorticity be accurately approximated, and that the algorithm be stable. The filament method can be shown to satisfy these three requirements by reliance on a particular coordinate transformation. This transformation is a map from a subset of $\mathbf{R}^{3}$ (which is periodic in one direction) to the support of the initial vorticity field such that the pre-images of vortex filaments are parallel lines. The images under this transformation of the nodes of a cubic grid serve as initial positions of fluid particles. Vortex stretching is evaluated by the application of a finite-difference operator along the filaments which is inherited through the coordinate transformation from a finite-difference approximation, on the cubic grid, to the partial derivative in the filamental direction. A formulation of the filament method is described in Section 2. The algorithm there defined is the subject of analysis of the following sections.

A version of the Stability Lemma of Beale and Majda [2] is presented in Section 3 (Lemma 3.1). A brief remark about the terminology is in order. The method of Beale and Majda, as they presented it, is a system of ordinary differential equations with both the particle positions and the values of vorticity as independent variables. Although this algorithm is identical to the system of ordinary differential equations in particle positions alone reformulated in [1] (where vorticity values are given as explicit functions of the set of particle positions), the notions of stability and consistency are slightly different. In fact, Lemma 3.1, though primarily a statement about the stability of the algorithm, is also used in the proof of consistency. 
The nodes of the cubic grid and the volumes of the cubes of the grid form, respectively, the nodes and weights of the trapezoidal rule integration formula. By requiring the coordinate transformation described above to be smooth, and by multiplying the integration weights by the Jacobian of the transformation, we obtain an accurate integration formula on the support of the initial vorticity field, with nodes which lie along filaments. The smoothness of the coordinate transformation also implies that the difference formula used to evaluate vortex stretching is as accurate as the difference scheme from which it is inherited. In Section 4, it is observed that the consistency of the filament method follows.

The lemma showing stability of the filament method is presented in Section 5. It is an immediate consequence of Lemma 3.1.

The convergence theorem is stated and proved in Section 6. The error estimate given there is a bound of $C\left(\delta^{p}+h^{r}+h^{l} \delta^{1-l}+\Delta t^{m}\right)$ on the discrete $L^{2}$-norm of the error in the position of the filaments. Here $h$ is approximately the initial distance between neighboring particles used to resolve the vortex filaments, $\delta$ is a smoothing parameter, and $\Delta t$ and $m$ are, respectively, the size of the time step and the order of accuracy in a discretization in time of the ordinary differential equations which form a spatial discretization of Euler's equations. The constant $C$ depends on the time interval in which convergence is proved and on the integers $m$ and $p, r, l \geqslant 4$. The proof of this convergence theorem follows from the stability and consistency lemmas in the same way as in [1].

Numerical calculations of the interaction of two vortex rings, using a vortex filament method with Lagrangian spatial discretization as described in this paper, are reported in [8].

We conclude this introduction by mentioning that one can evaluate vortex stretching locally, by an explicit differentiation of the computational velocity field, rather than by finite differencing ([1], [12]). The filament method, however, still seems to be a better choice for inviscid calculations, while the local alternative is important because viscous effects can be incorporated into the algorithm by random walking (see [1] for further discussion comparing the two alternatives for evaluating vortex stretching).

2. The Vortex Filament Method. We introduce a vortex filament method in this section for which convergence obtains. We begin by recalling Euler's equations and then reformulate them in such a way that our filament method suggests itself as a natural and accurate discretization.

Let $\eta: \mathbf{R}^{3} \rightarrow \mathbf{R}^{3}$ be a smooth, divergence-free, and compactly supported vector field, and let $[0, T]$ be an interval of time on which a smooth solution to Euler's equations exists with $\eta$ as initial condition on the vorticity $\omega: \mathbf{R}^{3} \times[0, T] \rightarrow \mathbf{R}^{3}[10]$. $\omega$ satisfies the system of equations, called the vorticity formulation of Euler's equations,

$$
\begin{gathered}
\omega(x, 0)=\eta(x), \\
\partial \omega / \partial t+(u \cdot \nabla) \omega=(\omega \cdot \nabla) u, \\
u=K * \omega .
\end{gathered}
$$


Here the velocity $u: \mathbf{R}^{3} \times[0, T] \rightarrow \mathbf{R}^{3}$ is the divergenceless vector field whose curl is $\omega, K$ is the matrix

$$
K(x)=\frac{1}{4 \pi}\left(\begin{array}{ccc}
0 & x_{3} /|x|^{3} & -x_{2} /|x|^{3} \\
-x_{3} /|x|^{3} & 0 & x_{1} /|x|^{3} \\
x_{2} /|x|^{3} & -x_{1} /|x|^{3} & 0
\end{array}\right),
$$

and $*$ denotes convolution.

The flow map $\Phi: \mathbf{R}^{3} \times[0, T] \rightarrow \mathbf{R}^{3}$ corresponding to the solution of (2.1)-(2.3) is defined by

$$
\begin{gathered}
\Phi(\alpha, 0)=\alpha, \\
\partial \Phi(\alpha, t) / \partial t=u(\Phi(\alpha, t), t) .
\end{gathered}
$$

We abbreviate $\Phi_{\alpha}(t)=\Phi(\alpha, t), u_{\alpha}(t)=u\left(\Phi_{\alpha}(t), t\right)$, and $\omega_{\alpha}(t)=\omega\left(\Phi_{\alpha}(t), t\right)$ and define the Lagrangian functions $\Phi(t), u(t)$, and $\omega(t)$ by setting $\Phi(t)(\alpha)=\Phi_{\alpha}(t)$, $u(t)(\alpha)=u_{\alpha}(t)$, and $\omega(t)(\alpha)=\omega_{\alpha}(t)$.

A consequence of Euler's equations, and a basis for the construction and analysis of three-dimensional vortex methods, is the well-known formula

$$
\omega_{\alpha}(t)=(D \Phi(t)(\alpha)) \cdot \eta(\alpha),
$$

where $D \Phi(t)$ is the derivative of $\Phi(t)$ with respect to the spatial variables. Equation (2.6) expresses the fact that vectors of vorticity along particle trajectories expand and contract in proportion to the stretching of the fluid in the direction of the vorticity.

Let $A$ denote the support of $\eta$. We make the following simplifying assumption on $\eta$ : There is a compact (though not necessarily connected) set $B \subset \mathbf{R}^{2}$, a diffeomorphism (a smooth, nonsingular bijection) $X: \tilde{A} \rightarrow A$, where $\tilde{A}=B \times[0,2 \pi]$ (with faces $B \times\{0\}$ and $B \times\{2 \pi\}$ identified), and a function $c: A \rightarrow \mathbf{R}$ such that

$$
\eta(X(\tilde{\alpha}))=c(X(\tilde{\alpha})) \partial_{\phi} X(\tilde{\alpha}),
$$

for $\tilde{\alpha}=(b, \phi) \in \tilde{A}$. Here $\partial_{\phi}$ denotes differentiation in the $\phi$ direction. Thus, for $b \in B$, the images under $X$ of the (closed) lines $\{b\} \times[0,2 \pi]$ are vortex filaments at time $t=0$, that is, integral curves of $\eta$.

We push forward the operator $\partial_{\phi}$ from $\tilde{A}$ to $A$, and so define an operator $\partial_{\theta}$ of differentiation along the filaments, by setting

$$
\partial_{\theta} \Psi(\alpha)=c(\alpha) \partial_{\phi}(\Psi \circ X)\left(X^{-1}(\alpha)\right)
$$

for functions $\Psi: A \rightarrow \mathbf{R}^{3}$. Observe that the operator $\partial_{\theta}$ is proportional to the magnitude of the initial vorticity field and that $\partial_{\theta} \Phi(t)(\alpha)$ is proportional to the stretching at time $t$ of the vortex filament which runs through $\alpha$ at time 0 . In fact,

$$
\begin{aligned}
\omega_{\alpha}(t) & =(D \Phi(t)(\alpha)) \cdot \eta(\alpha)=c(\alpha)(D \Phi(t)(\alpha)) \cdot \partial_{\phi} X\left(X^{-1}(\alpha)\right) \\
& =c(\alpha) \partial_{\phi}(\Phi(t) \circ X)\left(X^{-1}(\alpha)\right)=\partial_{\theta} \Phi(t)(\alpha) .
\end{aligned}
$$

Define

$$
U[\Phi(t), \omega(t)](\alpha)=\int_{A} K\left(\Phi_{\alpha}(t)-\Phi_{\beta}(t)\right) \omega_{\beta}(t) d \beta
$$


By a change of variables in the integral (2.9), it can be verified that $U[\Phi(t), \omega(t)](\alpha)$ $=u_{\alpha}(t)$. Recalling Eqs. (2.4), (2.5), (2.8), we write Euler's equations in the form

$$
\begin{gathered}
\Phi_{\alpha}(0)=\alpha, \\
\partial \Phi(\alpha, t) / \partial t=U\left[\Phi(t), \partial_{\theta} \Phi(t)\right](\alpha) .
\end{gathered}
$$

A discretization in space of the system of equations (2.10)-(2.11), resulting in a system of ordinary differential equations, will now be described. Inspection of Eq. (2.11) shows that a discretization of the integral which defines $U$ and an approximation to the vorticity $\partial_{\theta} \Phi$ are required. A suitable system of finitely many equations can be obtained by following the positions of a finite number of particles, with Lagrangian coordinates which we denote by $\alpha_{i}$. The positions $\alpha_{i}$ will be chosen to lie along initial vortex filaments, so that the vorticity $\partial_{\theta} \Phi$ can be approximated by a finite-difference operator, and with the filaments to be resolved so spaced that the integral in (2.9) is accurately approximated by a finite summation.

We denote by $h$ a spatial discretization parameter, which we take to be of the form $h=2 \pi / m$, where $m$ is a positive integer. For $i=\left(i_{1}, i_{2}, i_{3}\right) \in \mathbf{Z}^{3}$, we set $\tilde{\alpha}_{i}=\left(b_{i}, \phi_{i}\right)$, where $b_{i}=h \cdot\left(i_{1}, i_{2}\right)$ and $\phi_{i}=h i_{3}$. The set $I(h)$, which is an index set for the system of ordinary differential equations corresponding to the discretization parameter $h$, is defined by

$$
I(h)=\left\{i \in \mathbf{Z}^{3}: h \cdot i \in \tilde{A}\right\}=\left\{i \in \mathbf{Z}^{3}: b_{i} \in B \text { and } 1 \leqslant i_{3} \leqslant m\right\} .
$$

Thus, the set of $\tilde{\alpha}_{i}$, for $i \in I(h)$, is the intersection of the set of nodes of a cubic grid of mesh-width $h$ with $\tilde{A}$. For these $i$, we set $\alpha_{i}=X\left(\tilde{\alpha}_{i}\right)$ and $p_{i}=h^{3}\left|D X\left(\tilde{\alpha}_{i}\right)\right|$, where $\left|D X\left(\tilde{\alpha}_{i}\right)\right|$ is the Jacobian of the transformation $X$ at $\tilde{\alpha}_{i}$. The $\alpha_{i}$ will be taken to be the initial positions of the fluid particles in the algorithm. Observe that the $\alpha_{i}$ corresponding to a fixed value of $b_{i}$ lie along one vortex filament, and that the set of nodes and weights $\left\{\alpha_{i}, p_{i}\right\}$, being the transformation of the set of nodes and weights of the trapezoidal rule, forms an integration formula on $A$ of infinite-order accuracy (see Section 4).

Vorticity is evaluated in the algorithm by a discrete approximation to the derivative of the flow map in the filamental direction. Let $\partial_{\phi}^{h}$ denote a discrete difference approximation (defined on the grid $\left\{\tilde{\alpha}_{i}\right\}$ ) to $\partial_{\phi}$, with $\partial_{\phi}^{h}$ defined appropriately near the $\phi=0$ and $\phi=2 \pi$ faces of $\tilde{A}$ in view of the partial periodicity of $\tilde{A}$. Then we define the approximation $\partial_{\theta}^{h}$ of $\partial_{\theta}$ by setting, for $\Psi:\left\{\alpha_{i} \in A\right\} \rightarrow \mathbf{R}^{3}$,

$$
\partial_{\theta}^{h} \Psi\left(\alpha_{i}\right)=c\left(\alpha_{i}\right) \partial_{\phi}^{h}(\Psi \circ X)\left(\tilde{\alpha}_{i}\right)
$$

We denote by $\tilde{\Phi}_{i}(t)$ the numerical approximation to $\Phi_{i}(t)=\Phi_{\alpha_{i}}(t)$, and, as before, let $\tilde{\Phi}(t):\left\{\alpha_{i} \in A\right\} \rightarrow \mathbf{R}^{3}$ be the map $\tilde{\Phi}(t)\left(\alpha_{i}\right)=\tilde{\Phi}_{i}(t)$. The vortex filament method whose convergence is proved in this paper consists of a numerical approximation of the solution of the following system of ordinary differential equations:

$$
\begin{gathered}
\tilde{\Phi}_{i}(0)=\alpha_{i}, \\
d \tilde{\Phi}_{i}(t) / d t=\tilde{U}\left[\tilde{\Phi}(t), \partial_{\theta}^{h} \tilde{\Phi}(t)\right]_{i},
\end{gathered}
$$

where $\tilde{U}$ is the discretization of (2.9) defined for $\Psi, \Omega:\left\{\alpha_{i} \in A\right\} \rightarrow \mathbf{R}^{3}$, by setting

$$
\tilde{U}[\Psi, \Omega]_{i}=\tilde{U}[\Psi, \Omega]\left(\alpha_{i}\right)=\sum_{j \in I(h)} K_{\delta}\left(\Psi_{i}-\Psi_{j}\right) \Omega_{j} p_{j}
$$


and $K_{\delta}$ is a member of a family, parametrized by $\delta$, of continuous approximations to $K$. The notation $\Psi_{i}=\Psi\left(\alpha_{i}\right), \Omega_{i}=\Omega\left(\alpha_{i}\right)$ has been employed.

Example. Consider a set of $k$ vortex rings $A_{1}, \ldots, A_{k}$, with cross sections identified with the pairwise disjoint sets $B_{1}, \ldots, B_{k} \subset \mathbf{R}^{2}$. The vortex rings may be unlinked or linked, knotted or not. Denote by $X_{m}$ the natural identification $X_{m}$ : $B_{m} \times[0,2 \pi] \rightarrow A_{m}$, for $m=1, \ldots, k$. Set

$$
A=\bigcup_{m=1}^{k} A_{m}, \quad B=\bigcup_{m=1}^{k} B_{m}, \quad \tilde{A}=B \times[0,2 \pi], \quad \text { and } \quad X=\bigcup_{m=1}^{k} X_{m}: \tilde{A} \rightarrow A
$$

Take $\partial_{\phi}^{h}$ to be the centered-difference operator $\partial_{\phi}^{h}(\Psi)\left(\tilde{\alpha}_{i}\right)=\left(\Psi_{i+\hat{\phi}}-\Psi_{i-\hat{\phi}}\right) / 2 h$, where $\hat{\phi}=(0,0,1)$. Observe that $|D X|=\left|\partial_{\phi} X\right|$. Thus,

$$
\begin{aligned}
\partial_{\theta}^{h} \tilde{\Phi}(t)\left(\alpha_{i}\right) p_{i} & =c\left(\alpha_{i}\right) \partial_{\phi}^{h}(\tilde{\Phi}(t) \circ X)\left(\tilde{\alpha}_{i}\right)\left|D X\left(\tilde{\alpha}_{i}\right)\right| h^{3} \\
& =\partial_{\phi}^{h}(\tilde{\Phi}(t) \circ X)\left(\tilde{\alpha}_{i}\right)\left|\eta\left(\alpha_{i}\right)\right| h^{3}=\left(\Phi_{i+\hat{\phi}}(t)-\Phi_{i-\hat{\phi}}(t)\right) \Gamma_{i},
\end{aligned}
$$

where $\Gamma_{i}=\left|\eta\left(\alpha_{i}\right)\right| h^{2} / 2$. Equation (2.13) then takes the more familiar looking form

$$
\frac{d}{d t} \tilde{\Phi}_{i}(t)=\sum_{j} K_{\delta}\left(\tilde{\Phi}_{i}(t)-\tilde{\Phi}_{j}(t)\right)\left(\tilde{\Phi}_{j+\hat{\phi}}(t)-\tilde{\Phi}_{j-\hat{\phi}}(t)\right) \Gamma_{j}
$$

See [5], for example, in which Chorin evaluates vortex stretching also by a centered-difference operator, though his operator is centered at the midpoints of the line segments joining adjacent particles, rather than at the particle positions themselves.

Assumptions on $K_{\delta}$. In order to prove sufficiently strong accuracy and stability results for convergence of the filament method to follow, certain assumptions need to be made on the smoothed kernels used in the algorithm. These conditions are the same as those given in [1] and are somewhat milder than those originally stated in [2]. We assume that for two integers $l, p \geqslant 4$, the kernels $K_{\delta}$ are of the form $K_{\delta}=K * f_{\delta}$, where $f_{\delta}(x)=\delta^{-3} f(x / \delta), \int_{\mathbf{R}^{3}} f=1, f \in C^{l}\left(\mathbf{R}^{3}\right)$, and $f$ satisfies the additional conditions:

(i) $\int_{\mathbf{R}^{3}} x^{\beta} f(x) d x=0$, for all multi-indices $\beta$ such that $1 \leqslant|\beta| \leqslant p-1$, $\int_{\mathbf{R}^{3}}|x|^{p}|f(x)| d x<\infty$

(ii) $|x|^{3+\beta}\left|D^{\beta} f(x)\right| \leqslant C$ for some $C$, and all $\beta$ such that $|\beta| \leqslant l$;

(iii) $|x|^{p+5}|f(x)| \leqslant C$ for some constant $C$.

A discussion, with examples, of functions $f$ satisfying these conditions is given in [4]. The functions $f$ and $f_{\delta}$ are often referred to as cutoff functions.

3. An Important Lemma. The lemma presented in this section, which is a modification of the Stability Lemma of Beale and Majda [2], is used both to prove stability and consistency of the filament method. Before stating the lemma, we define some norms.

For functions $\Psi:\left\{\alpha_{i} \in A\right\} \rightarrow \mathbf{R}^{3}$ and $\gamma:\left\{\tilde{\alpha}_{i} \in \tilde{A}\right\} \rightarrow \mathbf{R}^{3}$, set

$$
\begin{aligned}
\|\Psi\|_{L_{h}^{2}(A)}= & \left(\sum_{i \in I(h)}\left|\Psi_{i}\right|^{2} p_{i}\right)^{1 / 2}, \quad\|\gamma\|_{L_{h}^{2}(\tilde{A})}=\left(\sum_{i \in I(h)}\left|\gamma_{i}\right|^{2} h^{3}\right)^{1 / 2}, \\
& \|\gamma\|_{H_{h}^{1}(\tilde{A})}=\left(\|\gamma\|_{L_{h}^{2}(\tilde{A})}+\left\|D^{+} \gamma\right\|_{L_{h}^{2}(\tilde{A})}\right)^{1 / 2}, \\
& \|\gamma\|_{H_{h}^{-1}(\tilde{A})}=\sup \left|\left(\sum_{i \in I(h)} \gamma_{i} \tilde{\gamma}_{i} h^{3}\right)\right| /\|\tilde{\gamma}\|_{H_{h}^{1}(\tilde{A})},
\end{aligned}
$$


where the supremum in the last definition is over all functions $\tilde{\gamma}$ defined on $I(h)$, $D^{+} \gamma$ is the discrete (forward-difference) gradient of $\gamma$ (extended by periodicity in the periodic direction), and the $p_{i}$ are the integration weights introduced in the previous section. Of course, the $L_{h}^{2}(A)$ and $L_{h}^{2}(\tilde{A})$-norms are equivalent in the sense that $\|\Psi \circ X\|_{L_{h}^{2}(\tilde{A})} \leqslant C_{1}\|\Psi\|_{L_{h}^{2}(A)} \leqslant C_{2}\|\Psi \circ X\|_{L_{h}^{2}(\tilde{A})}$, with $C_{1}, C_{2}$ independent of $h$ and $\Psi$.

Lemma 3.1. There is a constant $C$ such that for all $t \in[0, T]$ and all $\Psi, \Omega$ : $\left\{\alpha_{i} \in A\right\} \rightarrow \mathbf{R}^{3}$ such that $\|\Psi-\Phi(t)\|_{L_{h}^{2}(A)} \leqslant \delta h^{3 / 2}$,

$$
\begin{aligned}
& \|\tilde{U}[\Psi, \Omega]-\tilde{U}[\Phi(t), \omega(t)]\|_{L_{h}^{2}(A)} \\
& \quad \leqslant C\left(\|\Psi-\Phi(t)\|_{L_{h}^{2}(A)}+\|(\Omega-\omega(t)) \circ X\|_{H_{h^{-1}(\tilde{A})}}\right) .
\end{aligned}
$$

We do not give the proof. Lemma 3.1 can be proved readily by minor modification of the proof of the Stability Lemma in [2], as we now discuss briefly. Recall that

$$
\begin{aligned}
\tilde{U}[\Psi, \Omega]_{i} & =\sum_{j \in I(h)} K_{\delta}\left(\Psi\left(\alpha_{i}\right)-\Psi\left(\alpha_{j}\right)\right) \Omega\left(\alpha_{j}\right) p_{j} \\
& =\sum_{j} K_{\delta}\left((\Psi \circ X)\left(\tilde{\alpha}_{i}\right)-(\Psi \circ X)\left(\tilde{\alpha}_{j}\right)\right)(\Omega \circ X)\left(\tilde{\alpha}_{j}\right) p_{j} .
\end{aligned}
$$

This sum is very similar to that studied in [2]. The three differences are that $X$, and hence $\Phi(t) \circ X$, is in general not measure preserving, the $p_{j}$ are not all identical $\left(=h^{3}\right.$ in [2]), and the space $\tilde{A}$ is periodic in one direction. These differences are of minor consequence. The first two lead to increases in the constant of the estimate by factors involving the maxima of the Jacobians of $X$ and of $X^{-1}$, which are bounded a priori. The estimate,

$$
\|g\|_{L^{\infty}(\tilde{A})} \leqslant C h^{-1 / 2}\left\|D^{+} g\right\|_{L^{2}(\tilde{A})},
$$

is used in the proof in [2] and is valid for grid functions $g$ having support contained within $\tilde{A}$. It can be replaced by the estimate $\|g\|_{L^{\infty}(\tilde{A})} \leqslant C h^{-1 / 2}\|g\|_{H_{h}^{1}(\tilde{A}) \text {, valid for }}$ periodic functions $g$, which follows from (3.1) by extending $g$ by periodicity and then multiplying $g$ by a cutoff function. The constant $C$ is of course increased.

It should be mentioned that a consequence of the restrictive hypothesis of Lemma 3.1 is that the convergence proof requires the condition $p \geqslant 4$ on the cutoff function, and thus does not apply to the so-called second-order kernels. Carrying out the whole convergence theory in $L^{q}$ (in place of $L^{2}$ ) spaces, however, with $q$ sufficiently large, one can show indirectly $L^{2}$-convergence of the algorithm even with $p=2$, as was done by Beale and Majda for their method [3] (and discussed in [1]).

4. Consistency. We study the accuracy of the filament method in this section. Thus, we seek a bound on the difference between the exact fluid velocities and the velocities calculated by the algorithm from the exact particle positions. Let $t \in[0, T]$ be fixed for the remainder of this section. The constants in the error bounds obtained below are independent of $t$ (but not of $T$ ). 
The consistency error estimate is naturally split into three parts. For the error in velocity at the $i$ th particle, we have

$$
\begin{aligned}
& \left|\tilde{U}\left[\Phi(t), \partial_{\theta}^{h} \Phi(t)\right]_{i}-u_{i}(t)\right| \\
& \leqslant\left|\tilde{U}\left[\Phi(t), \partial_{\theta}^{h} \Phi(t)\right]_{i}-\tilde{U}[\Phi(t), \omega(t)]_{i}\right| \\
& \quad+\left|\tilde{U}[\Phi(t), \omega(t)]_{i}-\int_{A} K_{\delta}\left(\Phi_{i}(t)-\Phi_{\beta}(t)\right) \omega_{\beta}(t) d \beta\right| \\
& \quad+\mid \int_{A} K_{\delta}\left(\Phi_{i}(t)-\Phi_{\beta}(T)\right) \omega_{\beta}(t) d \beta \\
& \quad-\int_{A} K\left(\Phi_{i}(T)-\Phi_{\beta}(t)\right) \omega_{\beta}(t) d \beta \mid .
\end{aligned}
$$

The first term on the right-hand side of Eq. (4.1) represents the difference between velocities computed at the exact particle positions using, on the one hand, the correct values of vorticity, and, on the other hand, the finite-difference approximation to the vorticity. The second term represents the difference between the velocity computed by the algorithm from the correct particle positions and correct vorticity values and the exact velocity smoothed by the cutoff function $f_{\delta}$. The final term is the difference between the exact velocity and its smoothing. We now proceed to consider these terms in turn and then present the consistency estimate (Lemma 4.5) needed for the convergence proof given in Section 6.

Assume that $\partial_{\phi}^{h}$ is $r$ th-order accurate. Then, since

$$
\begin{aligned}
\left|\partial_{\theta}^{h} \Phi(t)\left(\alpha_{i}\right)-\omega_{i}(t)\right| & =\left|\partial_{\theta}^{h} \Phi(t)\left(\alpha_{i}\right)-\partial_{\theta} \Phi(t)\left(\alpha_{i}\right)\right| \\
& =\left|c\left(\alpha_{i}\right)\right|\left|\left(\partial_{\phi}^{h}-\partial_{\phi}\right)(\Phi(t) \circ X)\left(\tilde{\alpha}_{i}\right)\right|,
\end{aligned}
$$

we conclude from the smoothness of $\Phi(t), X$, and $c$ that there is a constant $C$ such that

$$
\left\|\partial_{\theta}^{h} \Phi(t)-\omega(t)\right\|_{H_{h}^{-1}(A)} \leqslant C h^{r} .
$$

Application of a simple case $(\Psi=\Phi(t))$ of Lemma 3.1 and Eq. (4.2) yields

LEMMA 4.1. If $\partial_{\phi}^{h}$ is of rth-order accuracy, then there is a constant $C$ such that

$$
\left\|\tilde{U}\left[\Phi(t), \partial_{\theta}^{h} \Phi(t)\right]-\tilde{U}[\Phi(t), \omega(t)]\right\|_{L_{h}^{2}(A)} \leqslant C h^{r} .
$$

The second term to be estimated is $E_{d}\left(\Phi_{i}(t)\right)$, where $E_{d}$ is the discretization error

$$
E_{d}(x)=\left|\int_{A} K_{\delta}\left(x-\Phi_{\alpha}(t)\right) \omega_{\alpha}(t) d \alpha-\sum_{j \in I(h)} K_{\delta}\left(x-\Phi_{j}(t)\right) \omega_{j}(t) p_{j}\right| .
$$

Observe that $E_{d}(x)$ is the error in the application of the intergration formula with nodes $\alpha_{i}$ and weights $p_{i}$ to the function $F(\alpha)=K_{\delta}(x-\Phi(t)(\alpha)) \omega(t)(\alpha)$. This integration formula is arbitrarily accurate, in the sense that for all integers $l \geqslant 4$, we have

Lemma 4.2. Let $F: A \rightarrow \mathbf{R}$ with $\operatorname{supp}(F) \subseteq A$. Then, for some constant $C$ independent of $h$,

$$
\left|\int_{A} F(\alpha) d \alpha-\sum_{i \in I(h)} F\left(\alpha_{i}\right) p_{i}\right| \leqslant C\|F\|_{W^{\prime \cdot 1}(A)} h^{\prime}
$$

where $\|F\|_{W^{\prime, 1}(A)}=\max _{\beta \leqslant l} \int_{A}\left|D^{\beta} F(\alpha)\right| d \alpha$. 
Proof. Let $G: \tilde{A} \rightarrow \mathbf{R}$ be the function $G=(F \circ X) \cdot|D X|$. It can be seen, by a change of variables, that

$$
\int_{A} F(\alpha) d \alpha=\int_{\tilde{A}} G(\tilde{\alpha}) d \tilde{\alpha}, \quad \sum_{i \in I(h)} F\left(\alpha_{i}\right) p_{i}=\sum_{i \in I(h)} G\left(\tilde{\alpha}_{i}\right) h^{3} .
$$

Thus, the quantity estimated in the lemma is the error in the application of the trapezoidal rule to the smooth function $G$, defined on $\tilde{A}$, which is periodic in one direction and vanishes at the boundaries in the other two directions. The arguments given in the proofs of Lemmas 9 and 12 by Cottet and Raviart [7] show that

$$
\left|\int_{\tilde{A}} G(\tilde{\alpha}) d \tilde{\alpha}-\sum_{i \in I(h)} G\left(\tilde{\alpha}_{i}\right) h^{3}\right| \leqslant C\|G\|_{W^{\prime, 1}(\tilde{A})} h^{\prime},
$$

for a constant $C$ independent of $G$ and $h$. The lemma now follows from this estimate and the assumed smoothness of $X$.

Lemma 4.3. There is a constant $C$ independent of $h$ and $\delta$, such that

$$
\left\|E_{d}\right\|_{L^{\infty}\left(\mathbf{R}^{3}\right)} \leqslant C h^{\prime} \delta^{1-l} .
$$

Proof. Fix $x \in \mathbf{R}^{3}$ and let $F$ be defined as above. Then

$$
\left|E_{d}(x)\right| \leqslant C\|F\|_{W^{\prime, 1}(A)} h^{\prime}
$$

for some $C$. A consequence of the hypotheses on the smoothing function $f$ is the estimate $\int_{A}\left|D^{\beta} K_{\delta}\left(x-\Phi_{\alpha}(t)\right)\right| d \alpha \leqslant C \delta^{1-l}$, for $|\beta| \leqslant l$ and all $x$ [1], which follows from the pointwise estimate $\left|D^{\beta} K_{\delta}(x)\right| \leqslant C(\max (x, \delta))^{-2-\beta}$ [2]. The a priori bounds on derivatives of $\Phi(t)$ and $\omega(t)$, together with the above absolute integral estimate on $D^{\beta} K_{\delta}$, yield

$$
\|F\|_{W^{\prime, 1}(A)} \leqslant C \delta^{1-l},
$$

with $C$ independent of $\delta, h$, and $x$, from which the lemma follows.

The final term on the right-hand side of Eq. (4.1) can be written as $E_{m}\left(\Phi_{i}(t)\right)$, where

$$
E_{m}(x)=\left|\left(K_{\delta} * \omega(t)\right)(x)-(K * \omega(t))(x)\right|
$$

is the error due to replacement of $K$ by $K_{\delta}$ in the integral formula which gives the velocity as a function of the vorticity. The special moment condition (i) on the cutoff function $f$, which was suggested by Beale and Majda for their vortex method [2], implies that $K_{\delta}$ is an accurate approximation to $K$, in the following sense.

LEMMA 4.4. There is a constant $C$, independent of $\delta$ and $h$, such that

$$
\left\|E_{m}\right\|_{L^{\infty}\left(\mathbf{R}^{3}\right)} \leqslant C \delta^{p} .
$$

The reader is referred to [1] or [2] for a proof.

The stability result used in the convergence proof being in the discrete $L^{2}$-norm, it is most useful to estimate the consistency error in this norm as well.

LEMma 4.5. For some constant $C$,

$$
\left\|\tilde{U}\left[\Phi(t), \partial_{\theta}^{h} \Phi(t)\right]-u(t)\right\|_{L_{h}^{2}(A)} \leqslant C\left(h^{\prime} \delta^{1-l}+\delta^{P}+h^{r}\right) .
$$


Proof. This result follows immediately from Lemmas 4.1, 4.3, 4.4 and the fact that for compact sets the discrete $L^{2}$-norm is bounded by a constant times the supremum norm.

5. Stability. Lemma 5.1 shows that the filament method is stable, in the sense that for sufficiently small errors in the computed trajectories, the discrete $L^{2}$-norm of the difference between the velocity computed from the exact particle positions and the velocity computed from the approximate particle positions is bounded by a constant multiple of the norm of the error in position.

LEMMA 5.1. There is a constant $C$, such that for all $t \in[0, T]$, and all $\Psi$ such that $\|\Phi(t)-\Psi\|_{L_{h}^{2}(A)} \leqslant \delta h^{3 / 2}$

$$
\left\|\tilde{U}\left[\Phi(t), \partial_{\theta}^{h} \Phi(t)\right]-\tilde{U}\left[\Psi, \partial_{\theta}^{h} \Psi\right]\right\|_{L_{h}^{2}(A)} \leqslant C\|\Phi(t)-\Psi\|_{L_{h}^{2}(A)} .
$$

Proof. By definition,

$$
\left(\partial_{\theta}^{h} \Phi(t)-\partial_{\theta}^{h} \Psi\right)\left(\alpha_{i}\right)=c\left(\alpha_{i}\right) \partial_{\phi}^{h}((\Phi(t)-\Psi) \circ X)\left(\tilde{\alpha}_{i}\right) .
$$

Thus, since the $c\left(\alpha_{i}\right)$ are bounded, we have from Lemma 3.1,

$$
\begin{aligned}
& \left\|\tilde{U}\left[\Phi(t), \partial_{\theta}^{h} \Phi(t)\right]-\tilde{U}\left[\Psi, \partial_{\theta}^{h} \Psi\right]\right\|_{L_{h}^{2}(A)} \\
& \quad \leqslant C\left(\|\Phi(t)-\Psi\|_{L_{h}^{2}(A)}+\left\|\left(\partial_{\theta}^{h} \Phi(t)-\partial_{\theta}^{h} \Psi\right) \circ X\right\|_{H_{h}^{-1}(\tilde{A})}\right) \\
& \leqslant C\left(\|\Phi(t)-\Psi\|_{L_{h}^{2}(A)}+\left\|\partial_{\phi}^{h}((\Phi(t)-\Psi) \circ X)\right\|_{H_{h}^{-1}(\tilde{A})}\right) \\
& \quad \leqslant C\left(\|\Phi(t)-\Psi\|_{L_{h}^{2}(A)}+\|(\Phi(t)-\Psi) \circ X\|_{L_{h}^{2}(\tilde{A})}\right) \leqslant C\|\Phi(t)-\Psi\|_{L_{h}^{2}(A)},
\end{aligned}
$$

where the third inequality above follows from the fact that, as for first-order differential operators between the continuous spaces, consistent difference operators $\partial_{\phi}^{h}$ give rise to bounded operators from $H_{h}^{-1}$ to $L_{h}^{2}$ (see [2]). The constants $C$ here are all different, of course.

6. Convergence. We are now ready to present the convergence theorem.

THEOREM. Assume that $\partial_{\phi}^{h}$ is an rth-order accurate centered-difference operator. Let $h, \delta$, and $\Delta t=T / n_{1}$ be sufficiently small, and assume $l \geqslant 4, r \geqslant 4$. Let $\tilde{\Phi}_{i}^{n}$ denote the approximation to $\tilde{\Phi}_{i}(n \Delta t)$ obtained from an integration of Eqs. (2.12)-(2.13) by a Runge-Kutta method of order $m=1$ or $m=2$, and set $\Phi_{i}^{n}=\Phi_{i}(n \Delta t)$. Let $C_{1}$ be the maximum of $\left\|\partial^{2} \Phi(t) / \partial t^{2}\right\|_{L^{\infty}(A)}$ and of the constants $C$ appearing in the statements of Lemma 4.5 and Lemma 5.1, and set $C_{2}=\exp \left(C_{1} T\right)-1$. Then

$$
\max _{0 \leqslant n \leqslant n_{1}}\left\|e^{n}\right\|_{L_{h}^{2}(A)} \leqslant C_{2}\left(\delta^{p}+h^{r}+h^{l} \delta^{1-l}+\Delta t^{m}\right),
$$

where $e_{i}^{n}=\tilde{\Phi}_{i}^{n}-\Phi_{i}^{n}$, provided $C_{2}\left(\delta^{p}+h^{r}+h^{l} \delta^{1-l}+\Delta t^{m}\right) \leqslant \delta h^{3 / 2}$.

The proof of the $m=2$ version of this theorem is very similar to the proof of Theorem 3.2 in [1], to which the reader is referred. We content ourselves here with giving the much shorter proof of the $m=1$ (Euler's method) case. Thus, we prove convergence of the algorithm

$$
\tilde{\Phi}_{i}^{0}=\alpha_{i}, \quad \tilde{\Phi}_{i}^{n+1}=\tilde{\Phi}_{i}^{n}+\Delta t \tilde{U}\left[\tilde{\Phi}^{n}, \partial_{\theta}^{h} \tilde{\Phi}^{n}\right]_{i} .
$$


Proof of Theorem (case $m=1$ ). The argument proceeds by induction. We will show that

$$
\left\|e^{n}\right\|_{L_{h}^{2}(A)} \leqslant C_{1} \Delta t\left(\sum_{j=0}^{n-1}\left(1+C_{1} \Delta t\right)^{j}\right)\left(\delta^{p}+h^{r}+h^{l} \delta^{1-l}+\Delta t\right)
$$

for all $n \leqslant n_{1}$, from which the theorem follows. Since $e_{i}^{0}=0$ for all $i,(6.1)$ is satisfied for $n=0$. Now assume that (6.1) is satisfied for some $n<n_{1}$, and set $t=n \Delta t$. Since $C_{1} \Delta t \sum_{j=0}^{n-1}\left(1+C_{1} \Delta t\right)^{j} \leqslant \exp \left(C_{1} T\right)-1=C_{2}$, it follows from (6.1) and the hypothesis on the parameters stated in the theorem, that

$$
\left\|e^{n}\right\|_{L_{h}^{2}(A)} \leqslant \delta h^{3 / 2} \text {. }
$$

This inequality permits application of the stability estimate Lemma 4.5 below. Since

$$
\begin{aligned}
e_{i}^{n+1} & =\tilde{\Phi}_{i}^{n+1}-\Phi_{i}^{n+1} \\
& =\tilde{\Phi}_{i}^{n}+\Delta t \tilde{U}\left[\tilde{\Phi}^{n}, \partial_{\theta}^{h} \tilde{\Phi}^{n}\right]_{i}-\left(\Phi_{i}^{n}+\Delta t u_{i}(t)+O\left(\Delta t^{2}\right)\right),
\end{aligned}
$$

we have

$$
\begin{aligned}
\left|e_{i}^{n+1}\right| \leqslant & \left|\tilde{\Phi}_{i}^{n}-\Phi_{i}^{n}\right|+\Delta t\left|\tilde{U}\left[\tilde{\Phi}^{n}, \partial_{\theta}^{h} \tilde{\Phi}^{n}\right]_{i}-u_{i}(t)\right|+C_{1} \Delta t^{2} \\
\leqslant & \left|e_{i}^{n}\right|+\Delta t\left|\tilde{U}\left[\tilde{\Phi}^{n}, \partial_{\theta}^{h} \tilde{\Phi}^{n}\right]_{i}-\tilde{U}\left[\Phi^{n}, \partial_{\theta}^{h} \Phi^{n}\right]_{i}\right| \\
& +\Delta t\left|\tilde{U}\left[\Phi^{n}, \partial_{\theta}^{h} \Phi^{n}\right]_{i}-u_{i}(t)\right|+C_{1} \Delta t^{2}
\end{aligned}
$$

The application of Lemmas 4.5 and 5.1 to the above inequality yields

$$
\left\|e^{n+1}\right\|_{L_{h}^{2}(A)} \leqslant\left(1+C_{1} \Delta t\right)\left\|e^{n}\right\|_{L_{h}^{2}(A)}+\Delta t C_{1}\left(\delta^{p}+h^{r}+h^{l} \delta^{1-l}+\Delta t\right),
$$

from which the $n+1$ case of Eq. (6.1) follows.

Remark 1. In practice, the filament method described in this paper is inadequate for the computation of all but the simplest flows, since in regions of large strain in the filamental direction, the computational elements get pulled far apart from each other, and it is necessary to interpolate new particles. It would be very interesting to obtain a convergence theorem which permits the interpolation of new points in the algorithm.

Remark 2. The convergence theorem as stated above assumes that the integration formula used in each "cross section" is the trapezoidal rule. In fact, any sufficiently accurate planar integration formula, in combination with the trapezoidal rule applied in the filamental direction, yields a convergent vortex method. For example, one can use a radial distribution of points when dealing with vortex rings of constant cross section.

Remark 3. As was pointed out in a similar situation in [2], the convergence of the particle trajectories implies convergence of the vorticity and of the interpolated velocity fields. Consider the approximate vorticity $\partial_{\theta}^{h} \tilde{\Phi}^{n}$, for example. Setting $\omega^{n}\left(\alpha_{i}\right)=\omega\left(\Phi_{i}^{n}, n \Delta t\right)$, we have

$$
\begin{aligned}
\left\|\omega^{n}-\partial_{\theta}^{h} \tilde{\Phi}^{n}\right\|_{H_{h}^{-1}(A)} & \leqslant\left\|\partial_{\theta} \Phi^{n}-\partial_{\theta}^{h} \Phi^{n}\right\|_{H_{h}^{-1}(A)}+\left\|\partial_{\theta}^{h}\left(\Phi^{n}-\tilde{\Phi}^{n}\right)\right\|_{H_{h}^{-1}(A)} \\
& \leqslant C h^{r}+C\left\|\Phi^{n}-\tilde{\Phi}^{n}\right\|_{L_{h}^{2}(A)} \leqslant C\left(\delta^{p}+h^{r}+h^{l} \delta^{1-l}+\Delta t^{m}\right) .
\end{aligned}
$$


Remark 4. Convergence estimates similar to those of the theorem, with arbitrarily high integers $m$ as exponents of $\Delta t$, can be given for a class of explicit multistep ordinary differential equation solvers of order $m$. See [1] for a similar proof of this assertion for the Beale and Majda algorithm.

Acknowledgment. This work is based on part of the author's $\mathrm{Ph} . \mathrm{D}$. thesis, written in the Mathematics Department at the University of California, Berkeley, under the supervision of Professor Alexandre Chorin, for whose invaluable guidance the author is very grateful. The author would also like to thank Professor Ole Hald, with whom he had a number of very helpful conversations about this work. A remark by Enrique Thomann was very valuable, as were discussions with Dr. Chris Anderson. Support by the Office of Naval Research under contract N00014-76-0-0316 at Berkeley and by the National Science Foundation under grant MCS-81-17526 at the Courant Institute is gratefully acknowledged.

Courant Institute of Mathematical Sciences

251 Mercer Street

New York University

New York, New York 10012

1. C. Anderson \& C. Greengard, "On vortex methods," SIAM J. Numer. Anal., v. 22, 1985, pp. 413-440.

2. J. T. Beale \& A. MAJDA, “Vortex methods. I: Convergence in three dimensions," Math. Comp., v. 39,1982, pp. 1-27.

3. J. T. Beale \& A. MajDa, "Vortex methods. II: Higher order accuracy in two and three dimensions," Math. Comp., v. 39, 1982, pp. 29-52.

4. J. T. BeAle \& A. Majda, "High order accurate vortex methods with explicit velocity kernels," $J$. Comput. Phys., v. 58, 1985, pp. 188-208.

5. A. J. ChORIN, "Estimates of intermittency, spectra, and blow-up in developed turbulence," Comm. Pure Appl. Math., v. 34, 1981, pp. 641-672.

6. G. H. Cottet, Méthodes Particulaires pour l'Équation d'Euler dans le Plan, Thèse de 3ème cycle, Université P. et M. Curie, Paris, 1982.

7. G. H. Cottet \& P. A. RAviART, "Particle methods for one-dimensional Vlasov-Poisson equations," SIAM J. Numer. Anal., v. 21, 1984, pp. 52-76. 1984.

8. C. Greengard, Three Dimensional Vortex Methods, Ph.D. Thesis, Univ. of California, Berkeley,

9. O. HALD, “The convergence of vortex methods, II," SIAM J. Numer. Anal., v. 16, 1979, pp. 726-755.

10. T. KATO, “Nonstationary flows of viscous and ideal fluids in $R^{3}$," J. Funct. Anal., v. 9, 1972, pp 296-305.

11. A. LEONARD, "Computing three-dimensional incompressible flows with vortex elements," Annual Review of Fluid Mechanics, v. 17, 1985, pp. 523-559.

12. P. A. Raviart, An Analysis of Particle Methods, CIME Course in Numerical Methods in Fluid Dynamics, Como, July 1983. 